\author{
Hajime Nawata $\cdot$ Senji Shirasawa $\cdot$ Naoki Nakashima \\ Eiichi Araki · Jun Hashiguchi · Seibei Miyake \\ Teruaki Yamauchi · Kazuyuki Hamaguchi \\ Hironobu Yoshimatsu · Haruo Takeda \\ Hideo Fukushima · Takayuki Sasahara \\ Kohei Yamaguchi · Noriyuki Sonoda - Tomoko Sonoda \\ Masahiro Matsumoto · Yoshiya Tanaka \\ Hidekatsu Sugimoto · Hirotaka Tsubouchi \\ Toyoshi Inoguchi · Toshihiko Yanase
}

Nakayasu Wake - Kenziro Narazaki - Takashi Eto Fumio Umeda · Mitsuhiro Nakazaki · Junko Ono Takashi Asano · Yasuko Ito · Shoichi Akazawa Iwaho Hazegawa · Nobuyuki Takasu Moritsugu Shinohara · Takeshi Nishikawa

Seiho Nagafuchi · Toshimitsu Okeda Katsumi Eguchi · Masanori Iwase · Mayuko Ishikawa Masayuki Aoki · Naoto Keicho - Norihiro Kato Kazuki Yasuda · Ken Yamamoto · Takehiko Sasazuki

\title{
Genome-wide linkage analysis of type 2 diabetes mellitus reconfirms the susceptibility locus on 11p13-p12 in Japanese
}

Received: 9 July 2004 / Accepted: 9 August 2004 / Published online: 14 October 2004

(C) The Japan Society of Human Genetics and Springer-Verlag 2004

\begin{abstract}
Type 2 diabetes mellitus is a heterogeneous disorder, and the development of type 2 diabetes mellitus is associated with both insulin secretion defect and insulin resistance. The primary metabolic defect leading to type 2 diabetes mellitus has been thought to be varied among populations, especially in Japanese and Caucasians. Here, we have done the genome-wide scan for type 2 diabetes mellitus using 102 affected Japanese sib-pairs to identify the genetic factors predisposing to type 2 diabetes mellitus. Nonparametric linkage analysis showed one suggestive evidence for linkage to $11 \mathrm{p} 13-$
\end{abstract}

H. Nawata $\cdot$ N. Sonoda $\cdot$ H. Tsubouchi $\cdot$ T. Inoguchi

T. Yanase $\cdot$ T. Eto

Department of Medicine and Bioregulatory Science,

Graduate School of Medical Sciences, Kyushu University,

Fukuoka, Japan

S. Shirasawa $\cdot$ M. Ishikawa

Department of Pathology, Research Institute,

International Medical Center, Tokyo, Japan

N. Nakashima

Department of Medical Informatics,

Kyushu University Hospital, Fukuoka, Japan

E. Araki · T. Nishikawa

Department of Metabolic Medicine,

Faculty of Medical and Pharmaceutical Sciences,

Kumamoto University, Kumamoto, Japan

J. Hashiguchi

Tenposan Clinic, Kagoshima, Japan

S. Miyake

Sasebo Central Hospital, Nagasaki, Japan

T. Yamauchi

Yukuhashi Central Hospital, Fukuoka, Japan

K. Hamaguchi $\cdot$ H. Yoshimatsu

Department of Internal Medicine 1,

Faculty of Medicine, Oita University, Oita, Japan p12 [D11S905: two-point maximum LOD score (MLS) of 2.89 and multipoint MLS of 2.32] and one nominally significant evidence for linkage to 6q15-q16 (D6S462: two-point MLS of 2.02). Interestingly, the 11p13-p12 region was reported to be a susceptibility locus for Japanese type 2 diabetes mellitus with suggestive evidence of linkage, and D11S905 was within $5 \mathrm{cM}$ to D11S935 with the highest MLS in the previous linkage analysis reported. The only overlapped susceptibility region with suggestive evidence of linkage for Japanese type 2 diabetes mellitus was D11S935-D11S905 among

\section{H. Takeda}

Yatsushiro General Hospital, Kumamoto, Japan

H. Fukushima

Public Tamana Central Hospital, Kumamoto, Japan

T. Sasahara

Omuta Tenryo Hospital, Fukuoka, Japan

K. Yamaguchi

Oita Prefectural Hospital, Oita, Japan

T. Sonoda

Sonoda Clinic, Kagoshima, Japan

M. Matsumoto

Kitakyushu Municipal Medical Center, Fukuoka, Japan

Y. Tanaka

First Department of Internal Medicine, School of Medicine,

University of Occupational \& Environmental Health,

Fukuoka, Japan

H. Sugimoto

Sugimoto Clinic, Fukuoka, Japan

N. Wake

National Health Insurance Takachiho Town Hospital,

Miyazaki, Japan

K. Narazaki

Narazaki Clinic, Fukuoka, Japan 
F. Umeda

Diabetology and Endocrinology Division of Internal Medicine,

Fukuoka Medical Association Hospital, Fukuoka, Japan

M. Nakazaki

Department of Cardiovascular,

Respiratory \& Metabolic Medicine

Graduate School of Medicine, Kagoshima University,

Kagoshima, Japan

J. Ono

Department of Laboratory Medicine,

School of Medicine,

Fukuoka University, Fukuoka, Japan

T. Asano

First Department of Internal Medicine,

School of Medicine,

Fukuoka University, Fukuoka, Japan

Y. Ito

Furugou Clinic, Oita, Japan

S. Akazawa

Shin-Koga Hospital, Fukuoka, Japan

I. Hazegawa

Kamiamakusa General Hospital, Kumamoto, Japan

N. Takasu

Department of Endocrinology and Metabolism, Faculty of Medicine, University of the Ryukyus, Okinawa, Japan

M. Shinohara

National Health Insurance Matsubase Town Hospital,

Kumamoto, Japan

S. Nagafuchi

Department of Medicine and Biosystemic Science,

Graduate School of Medical Sciences,

Kyushu University, Fukuoka, Japan

T. Okeda

Shin-Kokura Hospital, Fukuoka, Japan

K. Eguchi

First Department of Internal Medicine,

Graduate School of Biomedical Sciences,

Nagasaki University, Nagasaki, Japan

M. Iwase

Department of Medicine and Clinical Science,

Graduate School of Medical Sciences,

Kyushu University, Fukuoka, Japan

M. Aoki $\cdot$ K. Yamamoto

Medical Institute of Bioregulation, Kyushu University, Fukuoka, Japan

N. Keicho $\cdot$ N. Kato $\cdot$ K. Yasuda

Research Institute,

International Medical Center of Japan,

Tokyo, Japan

T. Sasazuki $(\square)$

International Medical Center of Japan, 1-21-1 Toyama,

Shinjuku-ku, Tokyo 162-8655, Japan

E-mail: sasazuki@nciryo.hosp.go.jp

Tel.: +81-3-32027181

Fax: $+81-3-52730113$

the three reports including this study. These results taken together suggest that a susceptibility gene for type 2 diabetes mellitus in Japanese will reside in 11p13-p12.
Keywords Type 2 diabetes mellitus $\cdot$ Japanese Affected sib-pairs $\cdot$ Linkage $\cdot$ Chromosome $11 p$

\section{Introduction}

Type 2 diabetes mellitus is one of the most common diseases, and its prevalence is dramatically increasing worldwide (Zimmet et al. 2001). Type 2 diabetes mellitus is a heterogeneous disorder, and the development of type 2 diabetes mellitus is associated with both insulin secretion defect and insulin resistance. Japanese patients with type 2 diabetes mellitus were reported to be characterized by a lower body mass index (BMI) and lower fasting insulin levels than other populations (Ehm et al. 2000). Insulin secretion defect is thought to be the primary defect in Japanese (Kadowaki et al. 1984) whereas impaired insulin sensitivity is the first metabolic defect predisposing to the development of type 2 diabetes mellitus in Caucasians (Martin et al. 1992). These findings suggest that Japanese individuals with type 2 diabetes mellitus will have a different genetic risk factor, which affects the responsiveness of insulin secretion to glucose, from other populations. Therefore, we need to identify the susceptibility genes for the development of type 2 diabetes mellitus in Japanese to start a primary prevention based on genetic information and to develop the personalized medicine for type 2 diabetes mellitus in Japanese. So far, two whole-genome linkage analyses were carried out using 224 affected sib-pairs (ASPs) from 159 Japanese families (Mori et al. 2002) and 256 ASPs from 164 Japanese families (Iwasaki et al. 2003), besides the analysis of 45 ASPs from 18 Japanese American families (Ehm et al. 2000). The Japanese people may have advantages in the genetic analysis of polygenic disorders like diabetes since they are supposed to be a relatively homogeneous population. However, the two previous reports on the ASP analysis in Japanese did not give good overlapping regions, except for $6 p$ and $2 q$, and it has been argued that the replication by the third panel is indispensable for genetic susceptibility loci in Japanese. Here, we have carried out the third whole-genome linkage analysis on 102 ASPs from 102 Japanese families to identify the susceptibility loci for the development of type 2 diabetes mellitus.

\section{Subjects and methods}

One hundred and two ASPs with type 2 diabetes mellitus from 102 families were collected mainly from the Kyushu region in southwestern Japan. Parents and other siblings were not available in this study. The participants were interviewed and examined and gave written informed consent. This project was approved by the ethics committees of the related institutes. The diagnosis of type 2 diabetes mellitus was made based on the American Diabetes Association's 1997 criteria (Expert 

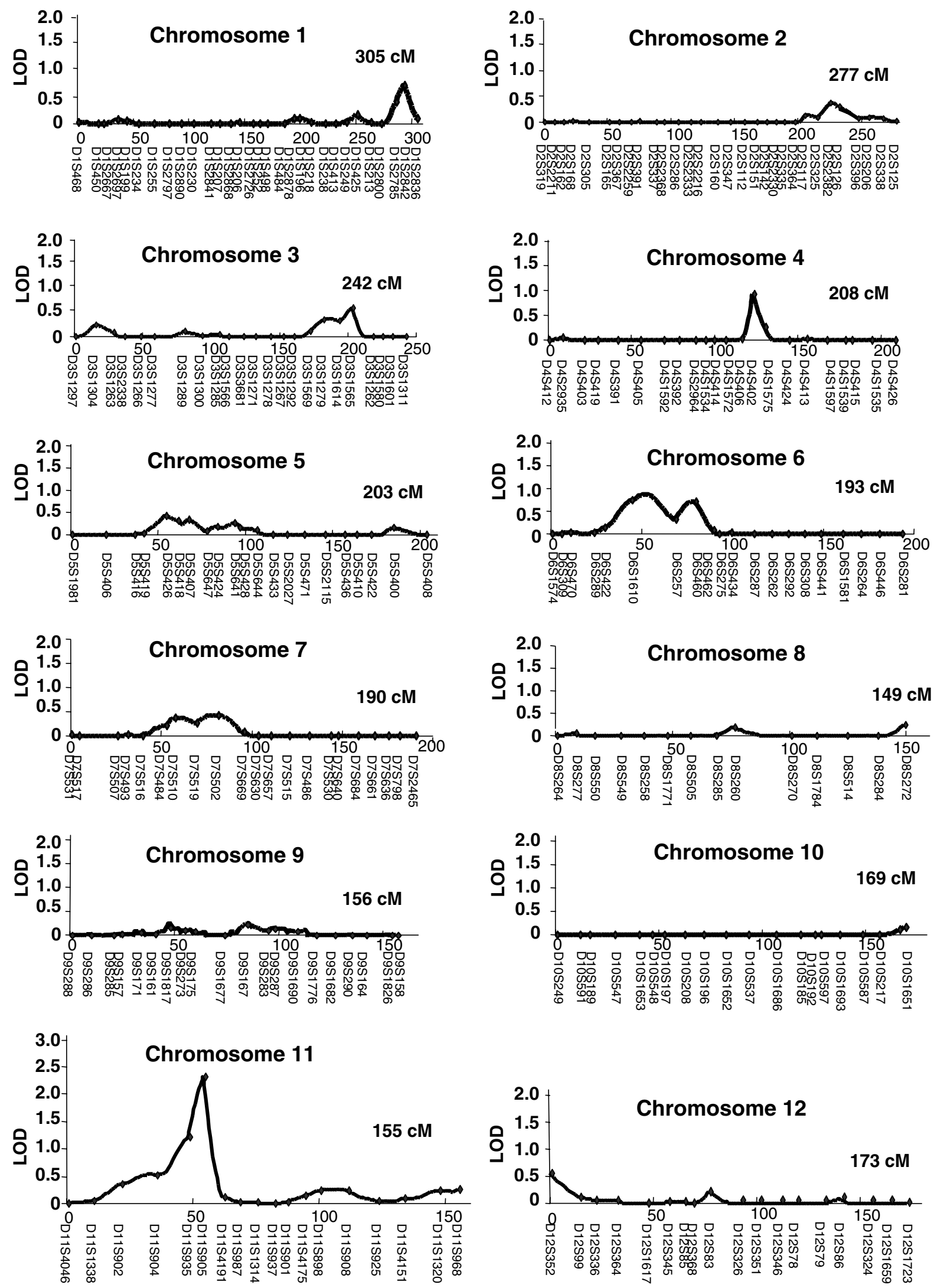

Fig. 1 Multipoint LOD score map of type 2 diabetes mellitus by linkage analysis of 382 markers in 102 affected sib-pairs. The horizontal axis is cM position from the p-terminal end of the chromosome 

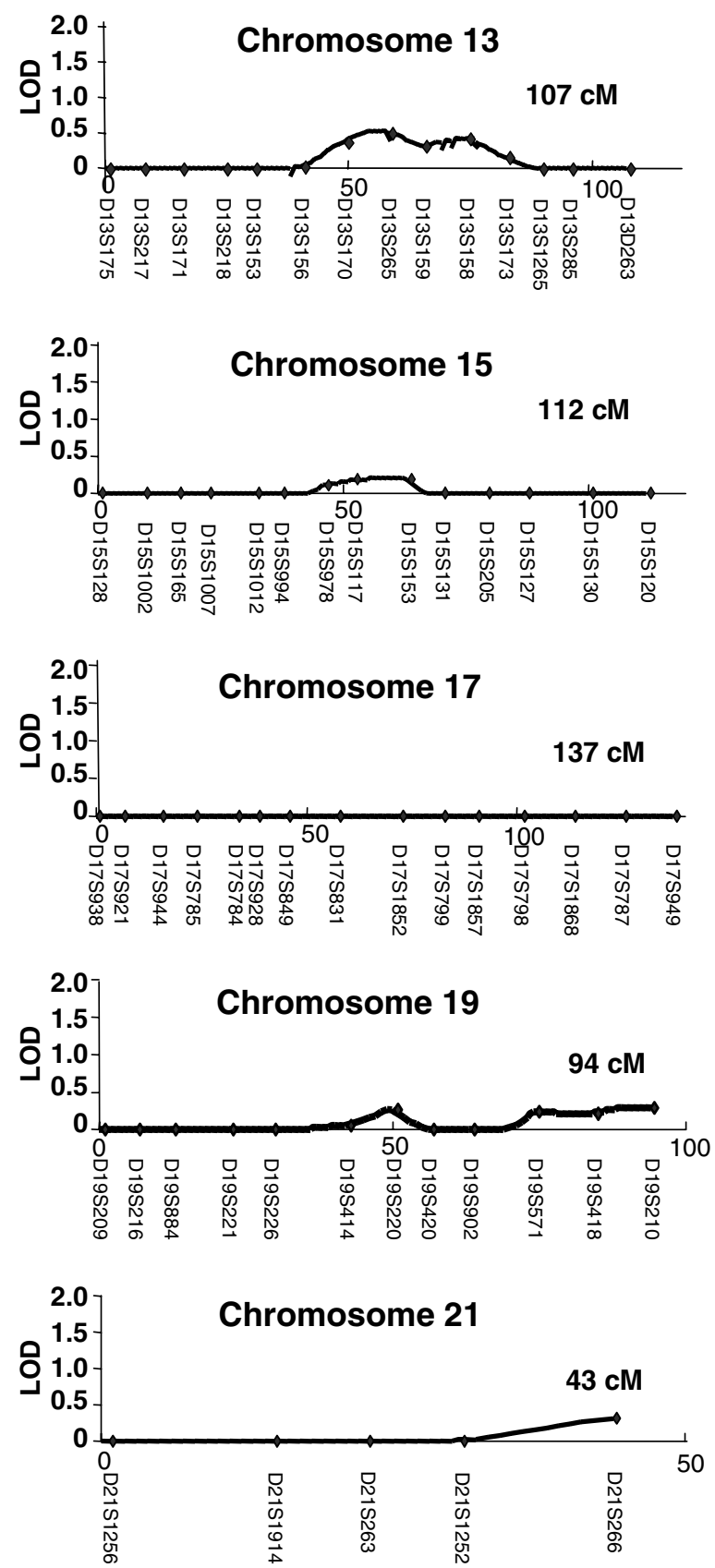

Fig. 1 (Continued)

Committee on the Diagnosis and Classification of Diabetes Mellitus 1997).

Genomic DNA was isolated from peripheral blood cells using QIAamp DNA Blood Midi Kits (Qiagen). Autosomal whole-genome screening of 382 microsatellite markers (ABI PRISM Linkage Mapping Set Version 2.5-MD10) was performed using an ABI 3730 automatic sequencer (Applied Biosytems). Analyses and assignment of the marker alleles were done with ABI PRISM GeneMapper Software Version 3.0, and 376 markers were available for the linkage analysis. Nonparametric two-point and multipoint linkage analyses
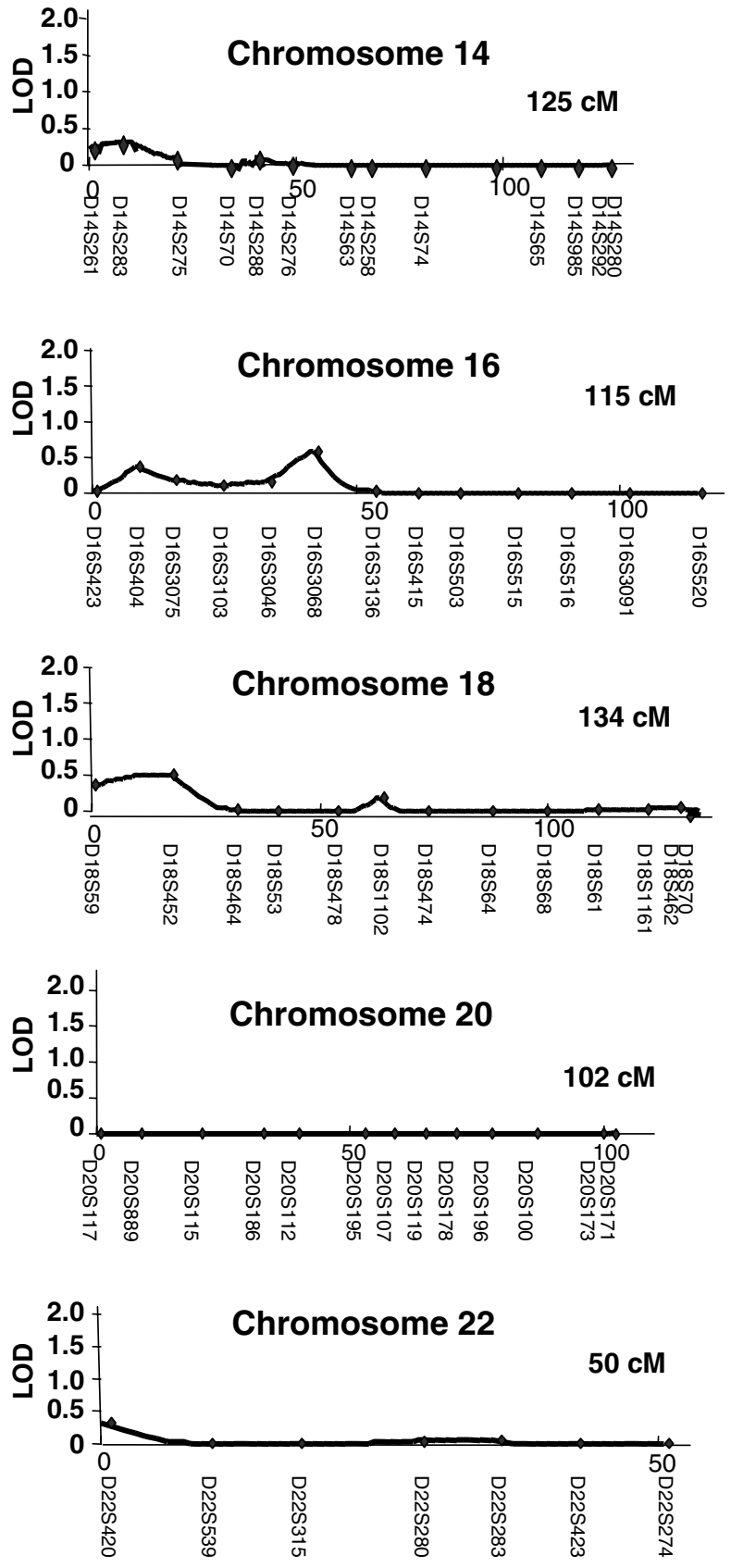

Table 1 Results of linkage analysis of type 2 diabetes mellitus and markers showing evidence of linkage

\begin{tabular}{lllll}
\hline Marker & $\mathrm{cM}^{\mathrm{a}}$ & Analysis & MLS & $P$ \\
\hline D6S462 & 89 & Two-point & 2.02 & 0.0097 \\
D11S905 & 54 & Two-point & 2.89 & 0.0013 \\
& & Multipoint & 2.32 & 0.0048
\end{tabular}

${ }^{\mathrm{a}}$ The distance of the marker form the p-terminal end of the chromosome in $\mathrm{cM}$

were performed with the MAPMAKER/SIBS program (Kruglyak and Lander 1995), as described (Sakai et al. 2001). Heterozygosities of the markers were estimated 
with Merlin program (Abecasis et al. 2002) for all individuals.

\section{Results and discussion}

Whole autosomal genome linkage analysis using the ASP method with 382 microsatellite markers was carried out on 102 Japanese ASPs with type 2 diabetes mellitus. In this study, the average heterozygosity of the markers used was 0.72 . Multipoint linkage analysis at all autosomal chromosomes using the MAPMAKER/SIBS program revealed only one region on chromosome $11 \mathrm{p}$ where the MLS was $>1$ (Fig. 1). The highest multipoint MLS was $2.32(P=0.0048)$ at D11S905 (Fig. 1, Table 1). On the other hand, two-point linkage analysis revealed two markers, D11S905 $(\mathrm{MLS}=2.89$, $P=0.0013$ ) and D6S462 (MLS $=2.02, P=0.0097$ ), with evidence of linkage to type 2 diabetes mellitus (Table 1). Although the heterozygosity of D11S905 was 0.30 in this study, it was 0.75 and 0.60 in our two reports using ASPs (Sakai et al. 2001; Aoki et al. 2004), indicating that D11S905 itself will be useful in the genetic analysis in terms of heterozygosity in the Japanese population, and particular alleles of D11S905 might be associated with type 2 diabetes mellitus. The $11 \mathrm{p} 13-\mathrm{p} 12$ region was reported to be linked to Japanese type 2 diabetes mellitus specifically, in which multipoint analysis showed the highest MLS of 3.08 near D11S935 (Mori et al. 2002). The distance between D11S905 and D11S935 is about $5 \mathrm{cM}$. These findings together suggest that the $11 \mathrm{p} 13-$ p12 region will be a susceptibility region for Japanese type 2 diabetes mellitus.

In addition to D11S905, one nominally significant evidence of linkage was detected at D6S462 (MLS of 2.02 ) by two-point analysis. However, the multipoint MLS at D6S462 was 0.08 , and two other reports did not show evidence of linkage to this region (Mori et al. 2002; Iwasaki et al. 2003), suggesting that 6q15-q16 might not be a susceptibility region for type 2 diabetes mellitus. Two susceptibility regions for type 2 diabetes mellitus in Japanese, chromosome $2(236.8 \mathrm{cM})$ and chromosome 6 $(42.2 \mathrm{cM})$, were reported to be overlapped between the two previous linkage studies (Iwasaki et al. 2003). However, the MLS at these two loci were $<1$ (Iwasaki et al. 2003). Among the three reports including this study, the overlapped susceptibility region with suggestive evidence of linkage for Japanese type 2 diabetes mellitus was D11S935-D11S905 only.

In conclusion, we have reconfirmed that the evidence of linkage for type 2 diabetes mellitus in Japanese to $11 \mathrm{p} 13-\mathrm{p} 12$ and $11 \mathrm{p} 13-\mathrm{p} 12$ will be a promising region for future studies on identification of susceptibility genes for type 2 diabetes mellitus in Japanese.

Acknowledgements We thank all patients for participating in this study, T. Amano in Kyushu University and T. Baba in SRL, Inc., for collecting the samples, and A. Murakami, E. Yachi, K. Ohki, and T. Fujimoto for technical assistance. This work was supported by the Program for Promotion of Fundamental Studies in Health Sciences of Pharmaceuticals and Medical Devices Agency (PMDA).

\section{Appendix}

Collaborating groups listed in alphabetic order: Nobuyuki Abe (Abe Diabetes Clinic), Katsumi Eguchi (Nagasaki University), Nobuaki Fujio (Beppu Medical Center), Masafumi Haji (Kyushu Rosai Hospital), Mine Harada (Kyushu University), Sinsuke Hiramatsu (Kyushu Medical Center), Mitsuo Iida (Kyushu University), Minako Imamura (Kyushu University), Hidehiro Ishii (Kitakyushu Municipal Medical Center), Eiji Kawasaki (Nagasaki University), Kunihisa Kobayashi (Kyushu University), Ichiro Komiya (University of the Ryukyus), Shiori Kondo (Matsuyama Red Cross Hospital), Yasuko Kono (Imamura Hospital), Nobuyuki Koriyama (Kagoshima University), Minoru Kuriyama (Social Health Insurance Inatsuki Hospital), Kazunari Matsumoto (Sasebo Central Hospital), Kazuo Mimura (Fukuoka Medical Association Hospital), Isao Morimoto (Inoue Hospital), Mieko Nakayama (Kyushu University), Shyoichi Natori (Aso Iizuka Hospital), Yosuke Okada (University of Occupational \& Environmental Health), Haruka Sasaki (Fukuoka University), Naotaka Sekiguchi (Kyushu University), Yasunori Sera (Sasebo Central Hospital), Michio Shimabukuro (University of the Ryukyus), Yuji Tajiri (Fukuoka Medical Association Hospital), Chuwa Tei (Kagoshima University), Maiko Tsuda (Kitakyushu Municipal Medical Center), Takero Uemura (Yatsushiro General Hospital), Eiji Kawasaki (Nagasaki University).

\section{References}

Abecasis GR, Cherny SS, Cookson WO, Cardon LR (2002) Merlin-rapid analysis of dense genetic maps using sparse gene flow trees. Nat Genet 30:97-101

Aoki M, Yamamura Y, Noshiro H, Sakai K, Yokota J, Kohno T, Tokino T, Ishida S, Ohyama S, Ninomiya I, Uesaka K, Kitajima M, Shimada S, Matsuno S, Yano M, Hiratsuka M, Sugimura $\mathrm{H}$, Itoh $\mathrm{F}$, Minamoto $\mathrm{T}$, Maehara $\mathrm{Y}$, Takenoshita $\mathrm{S}$, Aikou T, Katai H, Yoshimura K, Takahashi T, Akagi K, Sairenji M, Yamamoto K, Sasazuki T (2004) A full genome scan for gastric cancer. J Med Genet (in press)

Ehm MG, Karnoub MC, Sakul H, Gottschalk K, Holt DC, Weber JL, Vaske D, Briley L, Kopf J, McMillen P, Nguyen Q, Reisman M, Lai EH, Joslyn G, Shepherd NS, Bell C, Wagner MJ, Burns DK, ADA GENNID Study Group (2000) Genomewide search for type 2 diabetes mellitus susceptibility genes in four American populations. Am $\mathbf{J}$ Hum Genet 66:1871-1881

Expert Committee on the Diagnosis and Classification of Diabetes Mellitus (1997) Report of the expert committee on the diagnosis and classification of diabetes mellitus. Diabetes Care 20:11831197

Iwasaki N, Cox NJ, Wang Y-Q, Schwaz PEH, Bell GI, Honda M, Imura M, Ogata M, Saito M, Kamatani N, Iwamoto Y (2003) Mapping genes influencing type 2 diabetes mellitus risk and BMI in Japanese subjects. Diabetes 52:209-213 
Kadowaki T, Miyake Y, Hagura R, Akanuma Y, Kajinuma H, Kuzuya N, Takaku F, Kosaka K (1984) Risk factors for worsening to diabetes in subjects with impaired glucose tolerance. Diabetologia 26:44-49

Kruglyak L, Lander ES (1995) Complete multipoint sib-pair analysis of qualitative and quantitative traits. Am J Hum Genet 57:439-454

Martin BC, Warram JH, Krolewski AS, Bergman RN, Soeldner JS, Kahan CR (1992) Lancet 340:925-929

Mori Y, Otabe S, Dina C, Yasuda K, Populaire C, Lecoeur C, Vatin V, Durand E, Hara K, Okada T, Tobe K, Boutin P, Kadowaki T, Froguel P (2002) Genome-wide search for type 2 diabetes mellitus in Japanese affected sib-pairs confirms susceptibility genes on 3q, 15q, and 20q and identifies two new candidate loci on $7 \mathrm{p}$ and $11 \mathrm{p}$. Diabetes 51:1247-1255

Sakai K, Shirasawa S, Ishikawa N, Ito K, Tamai H, Kuma K, Akamizu T, Tanimura M, Furugaki K, Yamamoto K, Sasazuki T (2001) Identification of susceptibility loci for autoimmune thyroid disease to 5q31-q33 and Hashimoto's thyroiditis to 8q23-q24 by multipoint affected sib-pair linkage analysis in Japanese. Hum Mol Genet 10:1379-1386

Zimmet P, Alberti KGMM, Shaw J (2001) Global and societal implications of the diabetes epidemic. Nature 414:782-787 